\title{
Optimal Decisions in a Single-Period Supply Chain with Price-Sensitive Random Demand under a Buy-Back Contract
}

\author{
Feng Wang ${ }^{1}$ and In-Chan $\mathrm{Choi}^{2}$ \\ ${ }^{1}$ Graduate School of Information Management and Security, Korea University, Seoul 136-713, Republic of Korea \\ ${ }^{2}$ School of Industrial Management Engineering, Korea University, Seoul 136-713, Republic of Korea \\ Correspondence should be addressed to In-Chan Choi; ichoi@korea.ac.kr
}

Received 14 January 2014; Revised 15 June 2014; Accepted 19 June 2014; Published 16 July 2014

Academic Editor: Jung-Fa Tsai

Copyright (c) 2014 F. Wang and I.-C. Choi. This is an open access article distributed under the Creative Commons Attribution License, which permits unrestricted use, distribution, and reproduction in any medium, provided the original work is properly cited.

\begin{abstract}
This paper studies a single-period supply chain with a buy-back contract under a Stackelberg game model, in which the supplier (leader) decides on the wholesale price, and the retailer (follower) responds to determine the retail price and the order quantity. We analytically investigate the decentralized retailer's optimal decision. Our results demonstrate that the retailer has a unique optimal simultaneous decision on the retail price and the order quantity, under a mild restriction on the demand distribution. Moreover, as it can be shown that the decentralized supply chain facing price-sensitive random demand cannot be coordinated with buy-back contract, we propose a scheme for the system to achieve Pareto-improvement. Theoretical analysis suggests that there exists a unique Pareto-equilibrium for the supply chain. In particular, when the Pareto-equilibrium is reached, the supply chain is coordinated. Numerical experiments confirm our results.
\end{abstract}

\section{Introduction}

Buy-back contracts have become very prevalent in many industries; see [1] for some practical examples. With a buyback contract, a supplier compensates a retailer for unsold items at the end of selling season with a full or partial refund. Such contracts are usually used to mitigate the retailer's risk of overstocking, caused by uncertain demand. In his benchmark paper, Pasternack [2] suggests that in a singleperiod supply chain with an exogenously set retail price, channel coordination can be achieved under the buy-back contract where the supplier compensates the retailer with a partial refund for unsold items.

Many studies on supply chain contracts consider the retail price as an exogenous factor with a fixed value (see, e.g., [35]). However, in reality the retail price often varies by market and is usually an important decision variable of the retailer. Over the years, there has been a growing interest in studying the contract models which are based on the framework of price-sensitive and random demand (see $[6,7]$ for a good review). Following this stream of research, in this paper we consider endogenous retail price in a single-period supply chain and study the decisions and performance of the supply chain with a buy-back contract. Buy-back contracts are commonly used in many industries such as fashion apparel, publishing, and cosmetics [8], and the retailers in such industries need to decide the retail price before the selling season.

There have been many studies on supply chain contracts with price-sensitive demand. Among those, a group of researches focus on buy-back contracts. Particularly, the decision-making problems of each supply chain member in decentralized case, especially under Stackelberg framework, have generated a great deal of interest. Emmons and Gilbert [9] investigate the properties of each member's optimal decision in a single-period supply chain under a buy-back contract, by assuming that the demand has a linear and multiplicative form and follows uniform distribution. They suggest that there exists a domain of wholesale price where both the supplier and the retailer can earn more profits with a buy-back contract than with a wholesale price contract. A. H.-L. Lau and H.-S. Lau [10] extend the study of Emmons and Gilbert by considering an additive demand form. They employ numerical approach to study the effect of demand uncertainty on the performance of the channel and on 
the decisions of each member in the channel. Later, following A. H.-L. Lau and H.-S. Lau's research, Yao et al. [11] contribute to the literature by investigating the effect of price-sensitivity factors on the performance and the decisions of the supply chain. A common point in the above studies is that analytic solutions of the members' optimal decisions in a Stackelberg game are hard to find. For this reason, solutions in these studies are mainly based on numerical approaches. Arcelus et al. [12] introduce to the buy-back model the existence of a secondary market where the supplier can sell the leftover items at a price exceeding the salvage value. They investigate the optimal policy of each member in the supply chain with different demand forms and present closed-form solutions for the decentralized retailer's decision. However, their solutions are only for uniformly distributed demand.

In this paper, we investigate the decision making of each member of a single-period supply chain with a buy-back contract under a Stackelberg game, where the supplier, as the leader, determines the wholesale price to the retailer, while the retailer, as the follower, responds to decide the retail price and the number of units to purchase from the supplier. We consider the price-sensitive random demand in two forms: (1) the multiplicative form, which is characterized by an isoprice-elastic function multiplied by a random variable, and (2) the additive form, which is modeled as a linear function plus a random variable. The two forms of price-sensitive demand have been adopted in [13-15]. For each demand form, we investigate the decentralized retailer's simultaneous decision on the retail price and the order quantity. Our main contributions are twofold as follows.

(i) We demonstrate that under a mild restriction on the demand distribution, the decentralized retailer has a unique optimal joint decision. In particular, we provide analytic solutions of the decentralized retailer's optimal decision. In contrast to those solutions in previous works that are obtained either through numerical approaches or under the assumption that the demand is uniformly distributed [9-12], our solution is in a closed-form and only imposes a mild restriction on the demand distribution.

(ii) We provide a unique Pareto-equilibrium solution for the decentralized supply chain. Specifically, by comparing the integrated system's optimal decision and the decentralized retailer's optimal decision, we show that the buy-back contract cannot coordinate the system when the demand is price-sensitive and random, which indicates that the system profit is not maximized in the decentralized situation and, therefore, there may exist the space for making Pareto-improvement for the system. By employing Nash bargaining theory, we show that there exists a unique Pareto-equilibrium, for which no further Pareto-improvement can be made for the system. The Pareto-equilibrium solution allows both members in the supply chain to obtain better profits than in the decentralized case and thus is welcomed by the members. Furthermore, we show that when the unique
Pareto-equilibrium is reached, the supply chain is coordinated.

The rest of the paper is organized as follows. Section 2 describes the model. Section 3 derives the decisions of the members with a multiplicative form demand. Section 4 studies the decisions with a linear and additive demand model. Section 5 proposes a Pareto-equilibrium scheme. Section 6 presents the numerical experiment and Section 7 concludes the whole paper.

\section{The Model}

Consider a single-period supply chain facing price-sensitive random demand with a buy-back contract under Stackelberg game. The supplier, as the leader, acquires the products at unit cost $\widehat{c}$ and delivers them to the retailer at unit wholesale price $\widehat{w}$. The retailer, as the follower, then responds to decide the order quantity $q$ and the retail price $\widehat{p}$ to the consumers. The unsold items at the end of the selling season are bought back from the retailer at per unit cost $b$ which is predetermined before the selling season and is known to the whole channel. We clarify here that in the setting of this paper, the supplier does not actually buy back the unsold items but instead compensates the retailer with unit value $b$. Consequently, the salvage value ( $\widehat{v}$ per unit) occurs in the retailer. To prevent the retailer from making profits out of buy-back transactions only, we assume that $b+\widehat{v} \leq \widehat{w}$. For simplicity, we also assume that the unsatisfied demand carries no penalty cost.

We consider the demand to be both random and pricesensitive, where the randomness of the demand is represented in either additive [16] or multiplicative [17] form. Let $d(p)$ be a deterministic function characterizing the price dependency of the demand and let $\epsilon$ be a parameter representing the randomness in the demand. Then, the multiplicative and additive forms of demand are given as

$$
D(p, \epsilon)=d(p) \cdot \epsilon, \quad D(p, \epsilon)=d(p)+\epsilon,
$$

respectively. We assume that $\epsilon$ is price-independent and has a cumulative distribution function $F(\cdot)$ and a probability density function $f(\cdot)$, where $F(\cdot)$ is continuous, strictly increasing, and differentiable with inverse cumulative distribution function $F^{-1}(\cdot)$, and $f(\cdot)$ is also continuous and differentiable. Moreover, we assume $\epsilon$ to be nonnegative and supported on $[A, B]$, where $0 \leq A<B$.

Let $S(q)=\mathbf{E}[\min (q, D(p, \epsilon))]$ and $I(q)=\mathbf{E}[(q-$ $\left.D(p, \epsilon))^{+}\right]=q-S(q)$ denote the expected sales quantity and the expected leftovers, respectively. Then, the expected profits of the supplier and retailer are expressed as

$$
\begin{aligned}
\pi_{S}(\widehat{w})=\widehat{w} q-\widehat{c} q-b I(q)=(\widehat{w}-\widehat{c}-b) q+b S(q), \\
\pi_{R}(\widehat{p}, q)=\widehat{p} S(q)+(b+\widehat{v}) I(q)-\widehat{w} q \\
=(\widehat{p}-b-\widehat{v}) S(q)-(\widehat{w}-b-\widehat{v}) q,
\end{aligned}
$$

respectively, and the integrated supply chain's expected profit becomes

$$
\Pi(\widehat{p}, q)=\pi_{S}(\widehat{p}, q)+\pi_{R}(\widehat{p}, q)=(\widehat{p}-\widehat{v}) S(q)-(\widehat{c}-\widehat{v}) q .
$$


For notational simplicity, define $w \equiv \widehat{w}-\widehat{v}, c \equiv \widehat{c}-\widehat{v}$ and $p \equiv \widehat{p}-\widehat{v}$. Then the profit functions can be rewritten as

$$
\begin{gathered}
\pi_{S}(w)=(w-c-b) q+b S(q), \\
\pi_{R}(p, q)=(p-b) S(q)-(w-b) q, \\
\Pi(p, q)=\pi_{S}(p, q)+\pi_{R}(p, q)=p S(q)-c q .
\end{gathered}
$$

Hereafter in this paper, unless otherwise specifically stated, $w, c$, and $p$ will denote the wholesale price, the procurement cost, and the retail price, respectively.

\section{Decisions of the Supply Chain with Iso-Price-Elastic and Multiplicative Demand Model}

In this section, we study the decisions of the supply chain with random and price-sensitive demand. As in $[6,12$, 13], we consider the multiplicative demand form which is characterized by an iso-price-elastic function of a random variable, that is,

$$
D(p, \epsilon)=d(p) \cdot \epsilon=\alpha p^{-\beta} \cdot \epsilon, \quad \alpha>0, \beta>1,
$$

where $\beta$ is the price-elasticity index of demand, which measures how sensitive the demand is to the change of the retail price. The larger the value of $\beta$, the more sensitive the demand is. We are particularly interested in $\beta>1$, which refers to the case of price-elastic products. ten as

From (7), the expected sales quantity $S(q)$ can be rewrit-

$$
S(q)=\mathbf{E}[\min (q, d(p) \cdot \epsilon)]=d(p)(z-\Lambda(z)),
$$

where $z \equiv q / d(p)$ is called the stocking factor of inventory [6] and $\Lambda(z)=\int_{A}^{z} F(x) d x$. By substituting (8) into (6), the integrated supply chain's profit function can be rewritten as

$$
\Pi(p, q)=\Pi(p, z)=d(p)(p z-c z-p \Lambda(z)) .
$$

Thus, to maximize the integrated supply chain profit, the optimal retail price $p^{*}$ and the optimal stocking factor $z^{*}$ for the product must be decided simultaneously.

In [13], it has been shown that the integrated supply chain has a unique optimal joint decision $\left(p^{*}, z^{*}\right)$ satisfying

$$
\begin{gathered}
F\left(z^{*}\right)=\frac{z^{*}+(\beta-1) \Lambda\left(z^{*}\right)}{\beta z^{*}}, \\
p^{*}=\frac{c \beta z^{*}}{(\beta-1)\left(z^{*}-\Lambda\left(z^{*}\right)\right)},
\end{gathered}
$$

provided that the demand distribution satisfies

$$
h(x)+\frac{x d h(x)}{d x}>0,
$$

where $h(x) \equiv f(x) /(1-F(x))$ is the failure rate of the demand distribution, which gives "the percentage decrease in the probability of a stock out from increasing the stocking quantity by one unit" [18]. This mild restriction (11) can be satisfied under an increasing failure rate (IFR) condition, which actually captures many distributions such as uniform distribution, normal distribution, gamma distribution (when shape parameter is no less than one), Weibull distribution (when shape parameter is no less than one), and beta distribution (when shape parameter is no less than one).

3.1. Decisions of the Decentralized Supply Chain. In the decentralized supply chain modeled as a Stackelberg game in this paper, the supplier and the retailer separately make their decisions to maximize their own profits. The supplier, as the leader, decides on the wholesale price of the product to the retailer, while the retailer, as the follower, responds to the supplier's offer to choose the retail price and order quantity.

3.1.1. Retailer's Decision. We analyze the decision making of the supplier and the retailer in a backward sequence by first investigating the retailer's decision. To maximize her own profit, the retailer simultaneously decides the retail price $p$ and the order quantity $q$ for the product. Denote $\left(p_{R}^{*}, q_{R}^{*}\right)$ as the optimal joint decision of the retailer. By substituting (8) into the retailer's profit function, we have

$$
\pi_{R}(p, z)=d(p)[p z-w z-(p-b) \Lambda(z)] .
$$

Thus, the problem of determining the optimal decision $\left(p_{R}^{*}, q_{R}^{*}\right)$ of the retailer is equivalent to choosing the optimal decision $\left(p_{R}^{*}, z_{R}^{*}\right)$.

Theorem 1. In the decentralized supply chain with a buy-back contract, the retailer has a unique optimal joint decision of the retail price and the order quantity satisfying

$$
\begin{gathered}
p_{R}^{*}=\frac{\beta}{\beta-1} \cdot \frac{z_{R}^{*} w-b \Lambda\left(z_{R}^{*}\right)}{z_{R}^{*}-\Lambda\left(z_{R}^{*}\right)}, \\
F\left(z_{R}^{*}\right)=\frac{z_{R}^{*} w+(w \beta-w-b \beta) \Lambda\left(z_{R}^{*}\right)}{z_{R}^{*} \beta(w-b)+b\left(z_{R}^{*}-\Lambda\left(z_{R}^{*}\right)\right)},
\end{gathered}
$$

provided that $h^{\prime}(x) \geq 0$

Proof. Our proof is analogically following the proof approach of Theorem 1 in [13]. We first find the optimal retail price for any fixed $z \in[A, B]$. The first partial derivative of (12) with respect to $p$ is given by

$$
\begin{aligned}
\frac{\partial \pi_{R}(p, z)}{\partial p}=\alpha p^{-\beta-1}[ & (p-b) \beta \Lambda(z) \\
& -(p-w) \beta z+p(z-\Lambda(z))]
\end{aligned}
$$

Because $\alpha p^{-\beta-1}>0$ for any $p>0, \partial \pi_{R}(p, z) / \partial p=0$ implies

$$
p_{R}^{*}(z)=\frac{\beta}{\beta-1} \cdot \frac{z w-b \Lambda(z)}{z-\Lambda(z)},
$$

which is the unique maximizer of $\pi_{R}(p, z)$, since $\partial \pi_{R}(p, z) /$ $\partial p>0$ for all $p<p_{R}^{*}(z)$ and $\partial \pi_{R}(p, z) / \partial p<0$ for all 
$p>p_{R}^{*}(z)$. By taking the first derivative of $p_{R}^{*}(z)$ with respect to $z$, we have

$$
\begin{aligned}
\frac{d p_{R}^{*}(z)}{d z} & =\frac{\beta(w-b)}{\beta-1} \cdot \frac{z F(z)-\Lambda(z)}{(z-\Lambda(z))^{2}} \\
& \geq \frac{\beta(w-b)}{\beta-1} \cdot \frac{z F(z)-\int_{A}^{z} F(z) d x}{(z-\Lambda(z))^{2}} \\
& =0,
\end{aligned}
$$

which indicates that $p_{R}^{*}(z)$ is increasing in $z$. In addition, we have

$$
p_{R}^{*}(A)=\frac{\beta}{\beta-1} \cdot \frac{A w-b \Lambda(A)}{A-\Lambda(A)}=\frac{\beta w}{\beta-1}>w .
$$

Thus, the optimal retail price $p_{R}^{*}(z)$ is indeed a feasible solution.

We next find the optimal stocking factor $z_{R}^{*}$ that maximizes $\pi_{R}\left(p_{R}^{*}(z), z\right)$. By the chain rule, we have

$$
\begin{aligned}
& \frac{d \pi_{R}\left(p_{R}^{*}(z), z\right)}{d z} \\
& =\frac{\partial \pi_{R}\left(p_{R}^{*}(z), z\right)}{\partial z}+\frac{\partial \pi_{R}\left(p_{R}^{*}(z), z\right)}{\partial p} \frac{d p^{*}(z)}{d z} \\
& =\alpha\left(p_{R}^{*}(z)\right)^{-\beta}\left[p_{R}^{*}(z)-w-\left(p_{R}^{*}(z)-b\right) F(z)\right] \\
& =\frac{\alpha\left(p_{R}^{*}(z)\right)^{-\beta}}{(\beta-1)(z-\Lambda(z))} \cdot G(z),
\end{aligned}
$$

where

$$
G(z) \equiv(z-\Lambda(z))(w-b F(z))+\beta(w-b)(\Lambda(z)-z F(z)) .
$$

Since the first factor in the third equation of (19) is always positive, the optimal stocking factor $z_{R}^{*}$ is required to satisfy $G(z)=0$, which gives us (14). With the facts that $G(A)=$ $w A>0, G(B)=-(w-b)(\beta-1)(B-\Lambda(B))<0$, and $G(z)$ is continuous, we know that $z_{R}^{*}$ exists in the interval $(A, B)$. Now, what remains to verify is the uniqueness of $z_{R}^{*}$. Observe that

$$
\begin{aligned}
G^{\prime}(z)= & (1-F(z))(w-b F(z))-f(z) \\
& \times[\beta z(w-b)+b(z-\Lambda(z))] \\
G^{\prime \prime}(z)= & -f(z)[w+2 b-3 b F(z)+\beta(w-b)] \\
& -f^{\prime}(z)[b(z-\Lambda(z))+\beta z(w-b)] \\
= & -h(z) G^{\prime}(z)-(1-F(z)) \\
& \times[b(z-\Lambda(z))+\beta z(w-b)] h^{\prime}(z) \\
& -f(z)[2 b(1-F(z))+\beta(w-b)] .
\end{aligned}
$$

Since $(1-F(z))[b(z-\Lambda(z))+\beta z(w-b)]>0$ and $f(z)[2 b(1-$ $F(z))+\beta(w-b)]>0$, it is clear that if $h^{\prime}(z) \geq 0$, then
$G^{\prime \prime}(z)<0$ at $G^{\prime}(z)=0$, which implies that $G(z)$ itself is a unimodal function. This, in conjunction with $G(A)>0$ and $G(B)<0$, guarantees the uniqueness of $z_{R}^{*}$ in $(A, B)$. We thus complete our proof.

Remark 2. Note that the condition $h^{\prime}(x) \geq 0$, known as the increasing failure rate (IFR), imposes very mild restriction on the demand distribution, as it can be satisfied by many distributions (e.g., uniform, normal, exponential, and gamma with parameter restrictions).

Remark 3. The decentralized supply chain is said to be coordinated if the system reaches the maximum channel profit. By comparing the decision $\left(p_{R}^{*}, z_{R}^{*}\right)$ of the decentralized retailer with the optimal decision $\left(p^{*}, z^{*}\right)$ of the integrated system, we find that $\left(p_{R}^{*}, z_{R}^{*}\right)=\left(p^{*}, z^{*}\right)$ holds only when $b=0$ and $w=c$. That is, the channel coordination is achieved only when $b=0$ and $w=c$. However, in this case, the supplier would have no incentive to carry any product, since his profit is zero. Therefore, we conclude that the decentralized supply chain with buy-back contract facing a multiplicative demand form cannot be coordinated.

3.1.2. Supplier's Decision. The supplier is to decide the wholesale price with a fixed buy-back rate to maximize his own expected profit. By substituting (8) into (5), the supplier's expected profit function is given by

$$
\pi_{S}(w)=d\left(p_{R}^{*}\right)\left[(w-c) z_{R}^{*}-b \Lambda\left(z_{R}^{*}\right)\right] .
$$

Noting that $p_{R}^{*}$ and $z_{R}^{*}$ are both functions of $w$, we take the first derivative of $\pi_{S}(w)$ to obtain

$$
\begin{aligned}
\frac{d \pi_{S}(w)}{d w}=\alpha\left(p_{R}^{*}\right)^{-\beta-1}\{ & -\beta\left[(w-c) z_{R}^{*}-b \Lambda\left(z_{R}^{*}\right)\right] \frac{d p_{R}^{*}}{d w} \\
& \left.+p_{R}^{*}\left[z_{R}^{*}+\left(w-c-b F\left(z_{R}^{*}\right)\right) \frac{d z_{R}^{*}}{d w}\right]\right\} .
\end{aligned}
$$

As it is difficult to analytically show the supplier's optimal decision on the wholesale price (even for the simple case of uniformly distributed demand), we employ numerical approach, which will be presented in Section 6 .

\section{Decisions of the Supply Chain with Linear and Additive Demand Model}

In this section, we study the decision of each member in the supply chain with an additive demand form. In particular, the demand is characterized by a linear function plus a random variable:

$$
D(p, \epsilon)=d(p)+\epsilon=\alpha-\beta p+\epsilon, \quad \alpha>0, \beta>0,
$$

where $\beta$ is the price elasticity representing the sensitivity of the demand to the retail price. With (24), the expected sales quantity $S(q)$ can be written as

$$
S(q)=\mathbf{E}[\min (q, d(p)+\epsilon)]=d(p)+z-\Lambda(z),
$$


where $z \equiv q-d(p)$, consistent with Thowsen [19], and $\Lambda(z)=\int_{A}^{z} F(x) d x$. By substituting (25) into the integrated supply chain profit function, we have

$$
\Pi(p, q)=\Pi(p, z)=(p-c)(z+d(p))-p \Lambda(z) .
$$

Thus, the problem of finding the optimal retail price and the optimal order quantity that maximize the integrated system profit is equivalent to finding the optimal retail price and the optimal stocking factor. In [14], it is shown that the integrated supply chain has a unique optimal joint decision $\left(p^{*}, z^{*}\right)$ satisfying

$$
p^{*}=\frac{\alpha+\beta c+z^{*}-\Lambda\left(z^{*}\right)}{2 \beta}, \quad F\left(z^{*}\right)=\frac{p^{*}-c}{p^{*}},
$$

provided that the demand distribution satisfies

$$
2 h^{2}(x)+\frac{d h(x)}{d x}>0
$$

where $h(x)=f(x) /(1-F(x))$. Note that condition (28) can be implied by the IFR condition.

4.1. Decisions of the Decentralized Supply Chain. As mentioned, each party of the decentralized supply chain independently makes his/her decision to maximize his/her own profit. We first investigate the retailer's decision and then the supplier's.

4.1.1. Retailer's Decision. By substituting (25) into the retailer's expected profit function, we have

$$
\pi_{R}(p, z)=(p-w)(z+d(p))-(p-b) \Lambda(z) .
$$

Thus, the problem of determining the optimal decision $\left(p_{R}^{*}, q_{R}^{*}\right)$ of the retailer is equivalent to choosing the optimal $\left(p_{R}^{*}, z_{R}^{*}\right)$. Note that the optimal order quantity $q_{R}^{*}=z_{R}^{*}+$ $d\left(p_{R}^{*}\right)$.

Theorem 4. With a buy-back contract, the decentralized retailer has a unique optimal joint decision of retail price and order quantity satisfying

$$
\begin{gathered}
p_{R}^{*}=\frac{\alpha+\beta w+z_{R}^{*}-\Lambda\left(z_{R}^{*}\right)}{2 \beta}, \\
F\left(z_{R}^{*}\right)=\frac{p_{R}^{*}-w}{p_{R}^{*}-b},
\end{gathered}
$$

provided that $h^{2}(x)+d h(x) / d x>0$.

Proof. First, for any fixed $z \in[A, B]$, we find the optimal retail price that maximizes $\pi_{R}(p, z)$. The first partial derivative of (29) with respect to $p$ is given by

$$
\frac{\partial \pi_{R}(p, z)}{\partial p}=\alpha-2 \beta p+\beta w+z-\Lambda(z) .
$$

Since $\partial^{2} \pi_{R}(p, z) / \partial p^{2}=-2 \beta<0$, the retailer's optimal retail price $p_{R}^{*}(z)$ is the unique root of $\partial \pi_{R}(p, z) / \partial p=0$; that is,

$$
p_{R}^{*}(z)=\frac{\alpha+\beta w+z-\Lambda(z)}{2 \beta} .
$$

Since $d p_{R}^{*}(z) / d z=(1-F(z)) /(2 \beta) \geq 0, p_{R}^{*}(z)$ is increasing in $z$. This, in conjunction with the fact that $p_{R}^{*}(A)>w$ (Since the demand is always positive, we have $D(w, A)=\alpha-\beta w+A>0$ and thus $\left.p_{R}^{*}(A)=(\alpha+\beta w+A) /(2 \beta)>(2 \beta w) /(2 \beta)=w\right)$, implies $p_{R}^{*}(z)>w$ for all $z \in[A, B]$. That is, the optimal retail price is always greater than the wholesale price, which validates the feasibility of the optimal retail price $p_{R}^{*}(z)$.

We then find the optimal stocking factor $z_{R}^{*}$ that maximizes $\pi_{R}\left(p_{R}^{*}(z), z\right)$. Notice that $\pi_{R}\left(p_{R}^{*}(z), z\right)$ is a function of $z$ only. By the chain rule, we have

$$
\begin{aligned}
\frac{d \pi_{R}\left(p_{R}^{*}(z), z\right)}{d z} & =\frac{\partial \pi_{R}\left(p_{R}^{*}(z), z\right)}{\partial z}+\frac{\partial \pi_{R}\left(p_{R}^{*}(z), z\right)}{\partial p} \frac{d p_{R}^{*}(z)}{d z} \\
& =p_{R}^{*}(z)-w-\left(p_{R}^{*}(z)-b\right) F(z) .
\end{aligned}
$$

Since $d \pi_{R}\left(p_{R}^{*}(z), z\right) /\left.d z\right|_{z=A}=p_{R}^{*}(A)-w>0$ and $d \pi_{R}\left(p_{R}^{*}(z)\right.$, $z) /\left.d z\right|_{z=B}=-w+b<0$ and also noting that $d \pi_{R}\left(p_{R}^{*}(z), z\right) / d z$ is continuous in $[A, B]$, we have that the optimal stocking factor $z_{R}^{*}$ exists in the interval $(A, B)$. Now, what remains to verify is the uniqueness of $z_{R}^{*}$.

From (34), we have

$$
\begin{aligned}
& \frac{d^{2} \pi_{R}\left(p_{R}^{*}(z), z\right)}{d z^{2}} \\
& =\frac{(1-F(z))^{2}}{2 \beta}-\left(p_{R}^{*}(z)-b\right) f(z) \\
& \quad=-\left(p_{R}^{*}(z)-b\right) f(z)\left[1-\frac{(1-F(z))^{2}}{2 \beta\left(p_{R}^{*}(z)-b\right) f(z)}\right] .
\end{aligned}
$$

Define $H(z)=f(z) /(1-F(z))^{2}$ and $T(z)=1-1 /\left[2 \beta\left(p_{R}^{*}(z)-\right.\right.$ b) $H(z)]$. Then,

$$
\frac{d^{2} \pi_{R}\left(p_{R}^{*}(z), z\right)}{d z^{2}}=-\left(p_{R}^{*}(z)-b\right) f(z) T(z) .
$$

Observe that

$$
\begin{aligned}
\frac{d H(z)}{d z} & =\frac{2 f^{2}(z)+f^{\prime}(z)(1-F(z))}{(1-F(z))^{3}} \\
& =\frac{1}{1-F(z)}\left[h^{2}(z)+\frac{d h(z)}{d z}\right]>0 .
\end{aligned}
$$

Because both $p_{R}^{*}(z)$ and $H(z)$ are strictly increasing in $z, T(z)$ is also strictly increasing in $z$.

Now, if $T(A) \geq 0$, then $T(z)>0$ for all $z \in(A, B)$, which implies that $d^{2} \pi_{R}\left(p_{R}^{*}(z), z\right) / d z^{2}<0$ for all $z \in(A, B)$. Thus, $\pi_{R}\left(p_{R}^{*}(z), z\right)$ is concave in $z$ and has a unique maximum value in $(A, B)$. For the alternative case where $T(A)<0$, since $T(B)=1$, there exists $z^{0} \in(A, B)$ such that $T\left(z^{0}\right)=0$, which implies that $T(z)<0$ for $z<z^{0}$ and $T(z)>0$ for $z>z^{0}$. That is, $\pi_{R}\left(p_{R}^{*}(z), z\right)$ is convex for $z<z^{0}$ and concave for $z>z^{0}$. This, together with the facts that $d \pi_{R}\left(p_{R}^{*}(z), z\right) /\left.d z\right|_{z=A}>0$ and $d \pi_{R}\left(p_{R}^{*}(z), z\right) /\left.d z\right|_{z=B}<0$, leads to the result that $\pi_{R}\left(p_{R}^{*}(z), z\right)$ is unimodal with a maximum value in the interval $(A, B)$. 
By combining the above two cases, we know that there exists a unique $z_{R}^{*} \in(A, B)$ that maximizes $\pi_{R}\left(p_{R}^{*}(z), z\right)$, given by (31).

Remark 5. The condition $h^{2}(x)+d h(x) / d x>0$ can be implied by IFR condition.

Remark 6. By comparing the decentralized retailer's optimal decision with the integrated system optimal strategy, we find that the channel coordination can be reached only when $b=0$ and $w=c$. However, this case may not be possible in practice since it implies that the suppliers have no profit. Therefore, we conclude that the buy-back contract does not coordinate the supply chain with additive demand form.

4.1.2. Supplier's Decision. The supplier decides on the wholesale price to maximize his own profit. His profit function is

$$
\pi_{S}(w)=(w-c)\left(z_{R}^{*}+d\left(p_{R}^{*}\right)\right)-b \Lambda\left(z_{R}^{*}\right) .
$$

Similar to the case of multiplicative demand form, it is difficult to give a closed-form solution of the supplier's optimal wholesale price for the case with additive demand form. Such a difficulty has been pointed out by some researchers. As an alternative, numerical method has been employed to analyze the supplier's decision. Readers are referred to [11, 12] for details.

\section{Pareto-Equilibrium of the Supply Chain}

Thus far, we have shown that when the demand is both random and price-dependent, the profit of the decentralized supply chain with buy-back contract is always lower than the optimal profit of the integrated system. Thus there may exist chances for the supply chain to make Pareto-improvement; that is, both the supplier and retailer are no worse off and at least one of them is better off.

Let $\pi_{d S}^{*}$ and $\pi_{d R}^{*}$ denote the optimal profit of the supplier and the retailer in the decentralized supply chain, respectively. Let $\pi_{p S}$ and $\pi_{p R}$ be Pareto-improved profit of the supplier and the retailer, respectively. Let $\Pi_{d}^{*}=\pi_{d S}^{*}+\pi_{d R}^{*}$ and $\Pi_{p}=\pi_{p S}+\pi_{p R}$ be the optimal channel profit in decentralized case and the Pareto-improved channel profit, respectively. Let $\Pi^{*}$ be the integrated system optimal profit. From the definition of Pareto-improvement, we have $\pi_{p S} \geq \pi_{d S}^{*}$ and $\pi_{p R} \geq \pi_{d R}^{*}$. In addition, we have $\Pi_{d}^{*}<\Pi_{p} \leq \Pi^{*}$. Define the decision set $K$ of Pareto-improvement as

$$
K=\left\{(w, p, z): \pi_{p S} \geq \pi_{d S}^{*}, \pi_{p R} \geq \pi_{d R}^{*}\right\} .
$$

Then, from the bargaining model introduced by Nash [20], an optimal bargaining payment scheme can be obtained by solving the following problem:

$$
\max _{(w, p, z) \in K}\left(\pi_{p S}-\pi_{d S}^{*}\right)\left(\pi_{p R}-\pi_{d R}^{*}\right) .
$$

The solution of the above problem is the Pareto-equilibrium solution, denoted as $\left(w_{p}^{*}, p_{p}^{*}, z_{p}^{*}\right)$. Let $\pi_{p S}^{*}$ and $\pi_{p R}^{*}$ be the profit of the supplier and the retailer obtained in Pareto-equilibrium condition, respectively. Then, we have the following theorem.
Theorem 7. The supply chain has a unique Pareto-equilibrium with

$$
\pi_{p S}^{*}=\frac{1}{2}\left(\Pi^{*}+\pi_{d S}^{*}-\pi_{d R}^{*}\right), \quad \pi_{p R}^{*}=\frac{1}{2}\left(\Pi^{*}+\pi_{d R}^{*}-\pi_{d S}^{*}\right) .
$$

The retail price, the stocking factor, and the wholesale price in Pareto-equilibrium status are given as

$$
z_{p}^{*}=z^{*}, \quad p_{p}^{*}=p^{*}, \quad w_{p}^{*}=\frac{\pi_{p S}^{*}}{q_{p}^{*}}+\left(1-\frac{S\left(q_{p}^{*}\right)}{q_{p}^{*}}\right) b+c,
$$

respectively. Moreover, the channel coordination is obtained when the Pareto-equilibrium is reached.

Proof. Note that $\Pi_{p}$ is the channel profit with Paretoimprovement. Then problem $(\mathrm{P})$ can be reformulated as

$$
\max _{(w, p, z) \in K}\left(\pi_{p S}-\pi_{d S}^{*}\right)\left(\Pi_{p}-\pi_{p S}-\pi_{d R}^{*}\right) .
$$

Since the objective function of $(\widehat{\mathrm{P}})$ can be rewritten as $-\pi_{p S}^{2}+$ $\left(\Pi_{p}+\pi_{d S}^{*}-\pi_{d R}^{*}\right) \pi_{p S}-\pi_{d S}^{*}\left(\Pi_{p}-\pi_{d R}^{*}\right), \pi_{p S}$ has a unique optimal solution with $\pi_{p S}=\left(\Pi_{p}+\pi_{d S}^{*}-\pi_{d R}^{*}\right) / 2$, regardless of the decision set. It is easy to see that the supplier's Paretoimproved profit $\pi_{p S}$ is increasing in $\Pi_{p}$, since $\pi_{d S}^{*}$ and $\pi_{d R}^{*}$ are both constants. In conjunction with the fact that $\Pi_{p}$ is bounded above by the integrated system optimal profit $\Pi^{*}$, we find that the optimal value of $\pi_{p s}$ is obtained when $\Pi_{p}$ achieves its optimal value $\Pi^{*}$. Similarly, the optimal value of $\pi_{p R}$ can be found as $\pi_{p R}^{*}=\left(\Pi_{p}+\pi_{d R}^{*}-\pi_{d S}^{*}\right) / 2$. Thus, we can come to a conclusion from problem $(\widehat{\mathrm{P}})$ that there are a unique $\pi_{p S}^{*}$ and $\pi_{p R}^{*}$. Moreover, the supply chain is coordinated when the Pareto-equilibrium is obtained. Since the integrated system optimal profit $\Pi^{*}$ can only be obtained when the retail price $p=p^{*}$ and the stocking factor $z=$ $z^{*}$, we have $p_{p}^{*}=p^{*}$ and $z_{p}^{*}=z^{*}$. Therefore, the only variable left to be determined in problem $(\widehat{\mathrm{P}})$ is $w_{p}^{*}$. With the supplier's optimal profit value in Pareto-equilibrium status (i.e., $\left.\pi_{p S}^{*}=\left(\Pi^{*}+\pi_{d S}^{*}-\pi_{d R}^{*}\right) / 2\right)$ and the supplier's profit function (i.e., $\left.\pi_{S}(p, q)=(w-c-b) q+b S(q)\right)$, we have that the optimal wholesale price in Pareto-equilibrium status is also unique and $w_{p}^{*}=\pi_{p S}^{*} / q_{p}^{*}+\left(1-S\left(q_{p}^{*}\right) / q_{p}^{*}\right) b+c$, where $q_{p}^{*}=q^{*}=d\left(p^{*}\right) z^{*}$ for the demand with a multiplicative form and $q_{p}^{*}=q^{*}=d\left(p^{*}\right)+z^{*}$ for the demand with an additive form.

Remark 8. The expression of the wholesale price $w_{p}^{*}$ is consistent with our intuition, since the first factor $\pi_{p S}^{*} / q_{p}^{*}$ is the average net profit from each item purchased by the retailer, the second factor $\left(1-S\left(q_{p}^{*}\right) / q_{p}^{*}\right) b$ describes the average buyback price that the supplier transfers to the retailer, and the last factor $c$ is the unit procurement cost.

Remark 9. Since both the supplier and the retailer are better off in the Pareto-equilibrium status, the Pareto-equilibrium 
solution is welcomed by both of the members who are risk neutral and profit-seeking.

\section{Numerical Experiment}

In this section, we present the results of numerical experiments to investigate the implications of the buy-back contract. Our main goals are (1) to show the optimal decision of each member in decentralized supply chain, (2) to show the Pareto-equilibrium result, and (3) to do sensitivity analysis by measuring the impacts of the price elasticity and the buy-back price on each decentralized party's decision and on the efficiency of the decentralized supply chain. Our experiments are performed with the multiplicative demand model. The experimental results of the additive demand form are presented in $[11,12]$. In our experiment, the values of the problem parameters are arbitrarily chosen solely for illustrative purpose: the unit procurement $\operatorname{cost} c=4$; the random variable $\epsilon$ follows a uniform distribution over $[0,100] ; \alpha=200$ and the price elasticity $\beta=1.5,2,2.5,3$; the buy-back parameter $b=0,1,2,3$. Since the price elasticity and the buy-back price have different values, there are 16 scenarios formed by all combinations of the parameters. For each scenario, we calculate the integrated system optimal solutions, the optimal solutions of each member in the decentralized supply chain under Stackelberg game, and the Pareto-equilibrium solutions. All the results are displayed in Table 1. The results are obtained by using Maple program version 14.00 on a $3.0 \mathrm{GHz}$ E8400 Windows 7 machine with 4 GB RAM.

Analysis of the results in Table 1 provides several interesting observations. First, by comparing the decision of the decentralized channel and that of the integrated system, we observe that the decentralized retailer always sets a higher retail price and orders fewer products; that is, $p_{R}^{*}>p^{*}$ and $q_{R}^{*}<q^{*}$ in each row. We also observe that the decentralized channel profit $\Pi_{d}^{*}$ is always lower than the system optimal profit $\Pi^{*}$. This confirms that the decentralized supply chain facing price-sensitive demand cannot be coordinated by buyback contract. Moreover, by comparing the performance of the decentralized system with that of the unique Paretoequilibrium, we find that both the supplier and the retailer are better off under the Pareto-equilibrium status; that is, $\pi_{p S}^{*}>\pi_{d S}^{*}$ and $\pi_{p R}^{*}>\pi_{d R}^{*}$ in each row. We also find that the whole channel is coordinated when the Pareto-equilibrium is reached. In the Pareto-equilibrium status, the wholesale price is always lower than that of the decentralized case; that is, $w_{p S}^{*}<w_{S}^{*}$ in each row.

Sensitivity analysis is conducted to study the effects of price elasticity and buy-back price on the optimal decisions of supply chain members and on the performance of the system. We first examine the impact of the price elasticity on the optimal solutions under each case. With a certain buy-back price $b$, by observing the values in the columns under decentralized system case, we find in our limited computational experiments that the wholesale price $w_{S}^{*}$, the retail price $p_{R}^{*}$, the order quantity $q_{R}^{*}$, the retailer's profit $\pi_{d R}^{*}$, and the supplier's profit $\pi_{d S}^{*}$ all decrease as the price-elasticity $\beta$ increases. Figures 1 and 2 show these phenomena under the scenario where $b=2$. The same behavior can be observed in the integrated and the Pareto-equilibrium cases. These findings indicate that high price-elastic product reduces the channel performance.

We then examine the impact of buy-back price by fixing the price elasticity. We observe in this particular example that the optimal wholesale price $w_{S}^{*}$, which is decided by the decentralized supplier, increases with the buy-back price $b$ and that the retail price $p_{R}^{*}$ and the order quantity $q_{R}^{*}$, decided by the decentralized retailer, increases and decreases, respectively, in the buy-back price $b$. Figure 3 shows the impact of buy-back price $b$ on the wholesale price $w_{S}^{*}$, the retail price $p_{R}^{*}$, and the order quantity $q_{R}^{*}$ under the scenario where $\beta=2$. We also observe that the optimal profits of both the supplier and the retailer in the decentralized channel both decrease with the buy-back price $b$. Figure 4 shows these phenomena under the scenario where $\beta=2$. We are rather surprised to find in this numerical example that the buy-back contract yields worse result than the wholesale price contract does. This result may only be applicable to this particular example. But it also tells that in some situations both the supplier and the retailer will choose not to adopt the buy-back contract compared with the wholesale price contract.

Finally, we examine the efficiency of the decentralized system which is represented by $\Pi_{d}^{*} / \Pi^{*}$. Since the decentralized supply chain facing price-dependent and random demand with buy-back contract cannot be coordinated, the efficiency is always lower than 1 . The numerical results in Table 1 show that the efficiency drops when the price elasticity increases with a fixed buy-back price. It also decreases when the buy-back price increases with a fixed price elasticity. This implies that the price elasticity and the buy-back price have significant impacts on the efficiency. We also analyze the profit share of the supplier in the decentralized case which is characterized by $\pi_{d S}^{*} / \Pi_{d}^{*}$. In addition to the finding that the supplier can obtain more first-mover advantage when the buy-back price increases, the numerical results also show that the supplier's profit share in the decentralized system rises when the price elasticity becomes larger.

\section{Conclusion}

In this paper, we studied a single-period supply chain with a buy-back contract under a Stackelberg game model. We considered two different forms of demand: (1) the iso-priceelastic and multiplicative form and (2) the linear and additive form. For each demand form, we analytically showed that under a very mild restriction on the demand distribution, the decentralized retailer has a unique optimal joint decision on the retail price and the order quantity. This amends the existing results which are obtained either from numerical analyses or under the assumption that the demand follows uniform distribution. Moreover, by comparing the decentralized retailer's optimal decision with the integrated system's optimal decision, we showed that the decentralized supply chain facing price-sensitive and random demand cannot be coordinated with the buy-back contract. As such, 


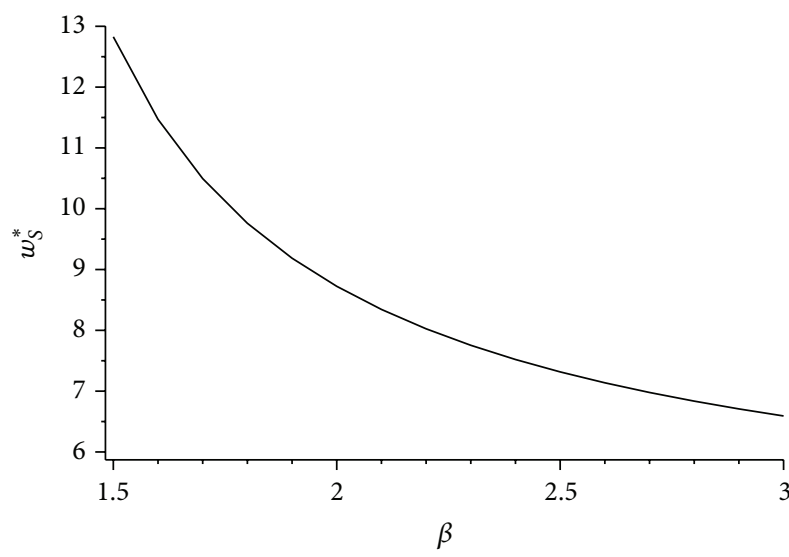

(a)

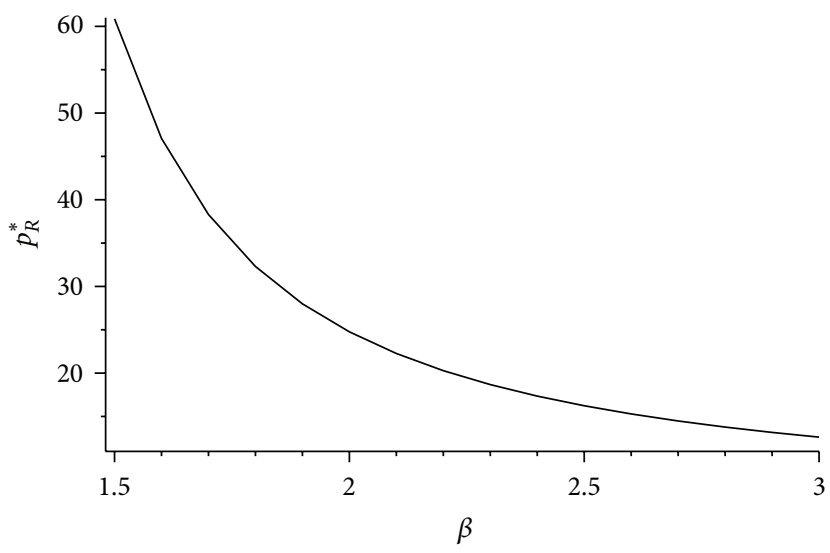

(b)

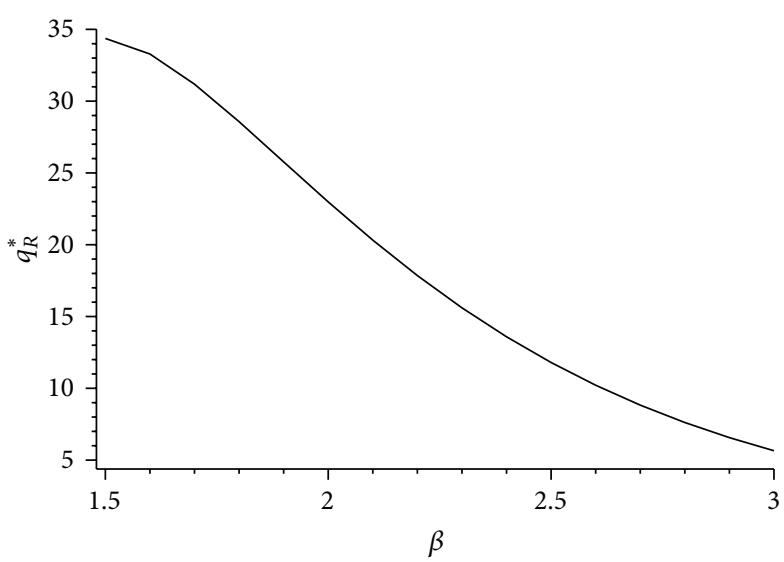

(c)

FIGURE 1: The impact of price elasticity $\beta$ on the wholesale price $w_{S}^{*}$, the retail price $p_{R}^{*}$, and the order quantity $q_{R}^{*}$ under the scenario where $b=2, c=4, D(p, \epsilon)=200 p^{-\beta} \cdot \epsilon, \epsilon \sim U[0,100]$. (a) The impact of price elasticity $\beta$ on the wholesale price $w_{S}^{*}$ when $b=2$. (b) The impact of price elasticity $\beta$ on the retail price $p_{R}^{*}$ when $b=2$. (c) The impact of price elasticity $\beta$ on the order quantity $q_{R}^{*}$ when $b=2$.

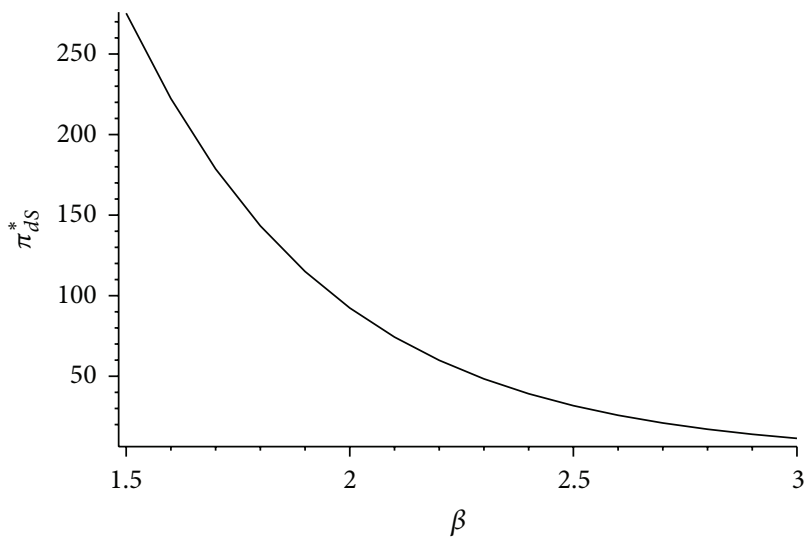

(a)

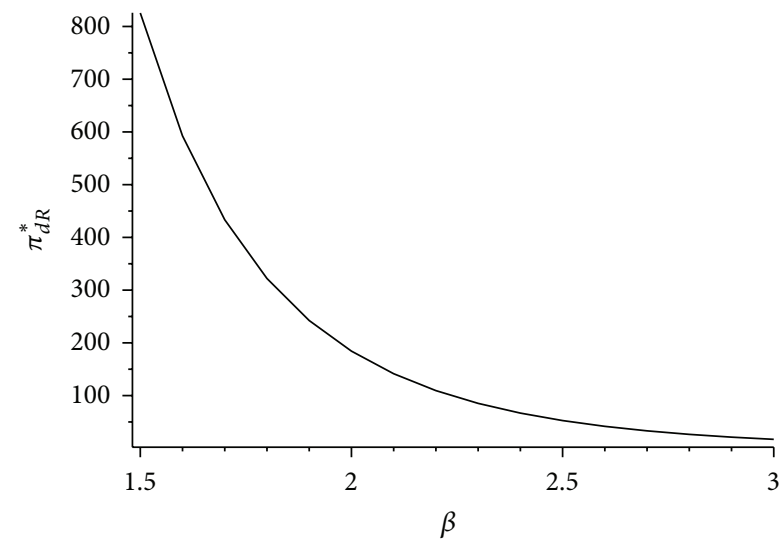

(b)

FIGURE 2: The impact of price elasticity $\beta$ on the decentralized supplier's profit $\pi_{d S}^{*}$ and the decentralized retailer's profit $\pi_{d R}^{*}$ under the scenario where $b=2, c=4, D(p, \epsilon)=200 p^{-\beta} \cdot \epsilon, \epsilon \sim U[0,100]$. (a) The impact of price elasticity $\beta$ on the decentralized supplier's profit $\pi_{d S}^{*}$ when $b=2$. (b) The impact of price elasticity $\beta$ on the decentralized retailer's profit $\pi_{d R}^{*}$ when $b=2$. 


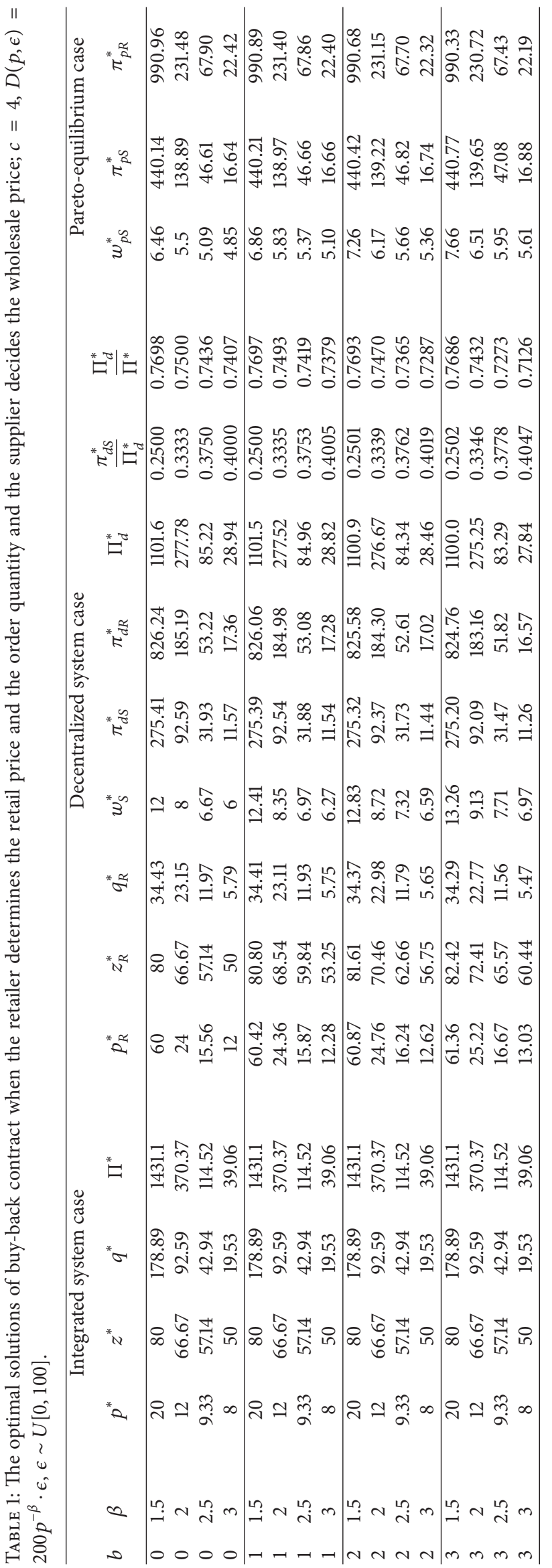




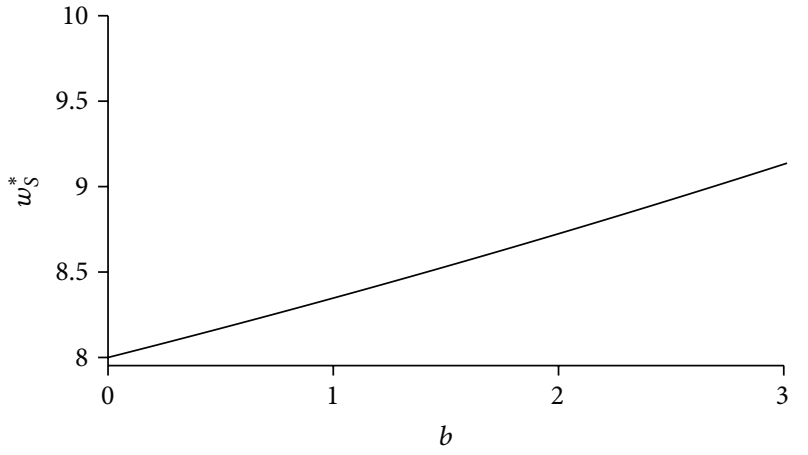

(a)

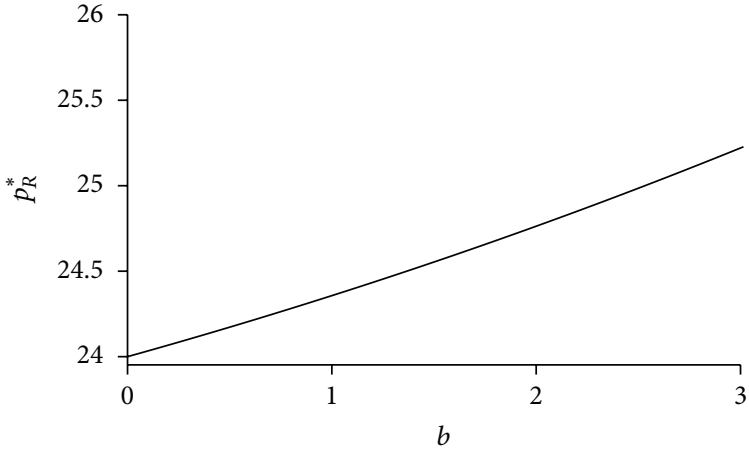

(b)

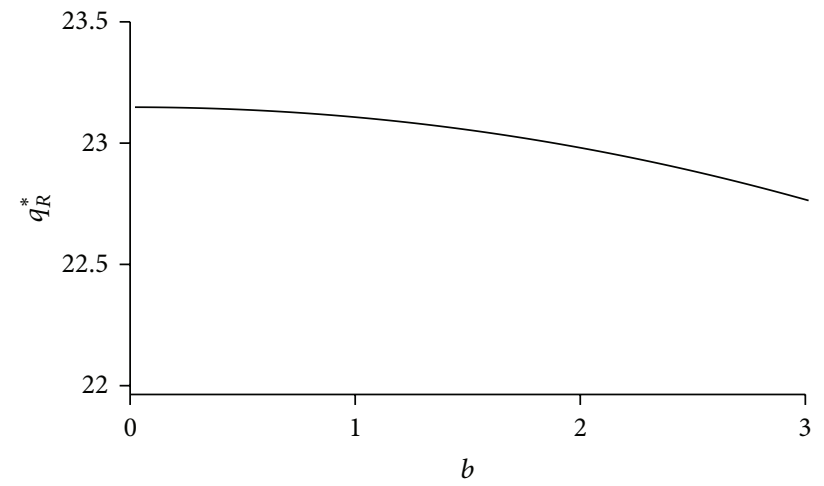

(c)

FIGURE 3: The impact of buy-back price $b$ on the wholesale price $w_{S}^{*}$, the retail price $p_{R}^{*}$, and the order quantity $q_{R}^{*}$ under the scenario where $\beta=2, c=4, D(p, \epsilon)=200 p^{-2} \cdot \epsilon, \epsilon \sim U[0,100]$. (a) The impact of buy-back price $b$ on the wholesale price $w_{S}^{*}$ when $\beta=2$. (b) The impact of buy-back price $b$ on the retail price $p_{R}^{*}$ when $\beta=2$. (c) The impact of buy-back price $b$ on the order quantity $q_{R}^{*}$ when $\beta=2$.

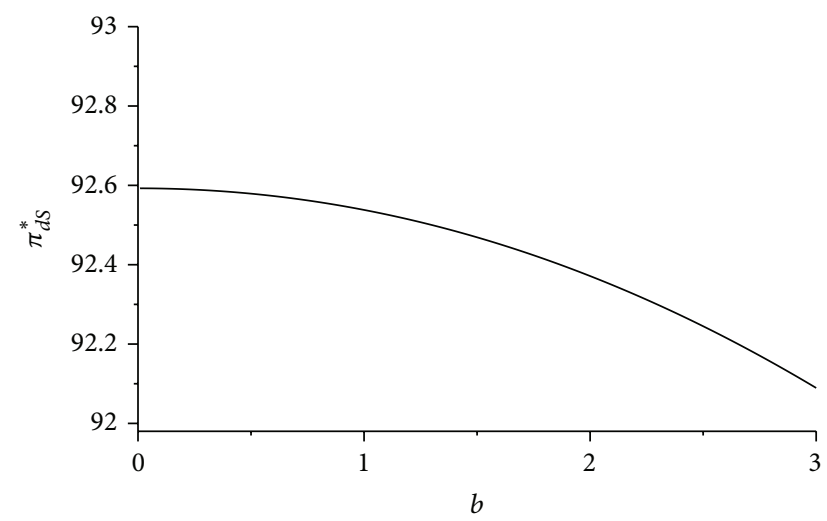

(a)

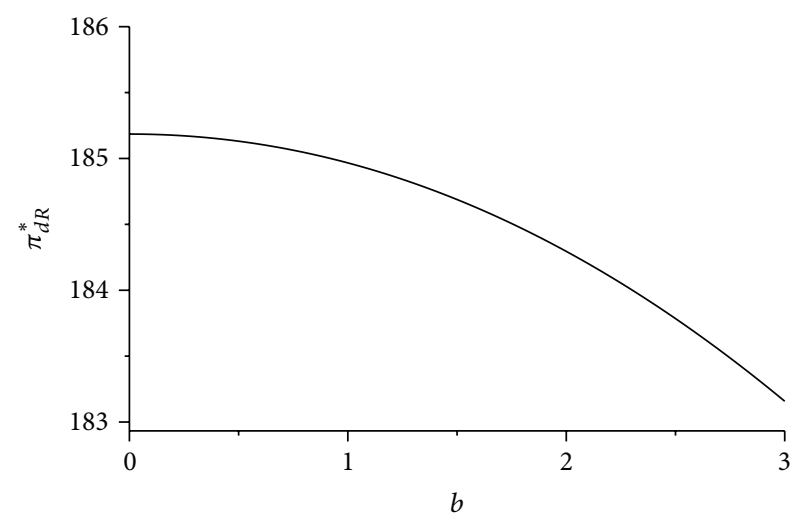

(b)

FIGURE 4: The impact of buy-back price $b$ on the decentralized supplier's profit $\pi_{d S}^{*}$ and the decentralized retailer's profit $\pi_{d R}^{*}$ under the scenario where $\beta=2, c=4, D(p, \epsilon)=200 p^{-2} \cdot \epsilon, \epsilon \sim U[0,100]$. (a) The impact of buy-back price $b$ on the decentralized supplier's profit $\pi_{d S}^{*}$ when $\beta=2$. (b) The impact of buy-back price $b$ on the decentralized retailer's profit $\pi_{d R}^{*}$ when $\beta=2$.

we analyzed the Pareto-improvement of the system. By employing a bargaining model, we showed that there exists a unique Pareto-equilibrium for the supply chain system. In particular, in the Pareto-equilibrium status, the supply chain is coordinated and the profit split scheme is unique, which highlight the managerial insight that a firm can be better off when coordinating with its counter partner.

Although in this paper we are primarily interested in the performance of the single-period supply chain with a single supplier and a single retailer, our results could be extended 
to the more general scenario where a supply chain has multiple periods and multiple members. Another possible extension of this study is to investigate "power relationship" and profit allocation in a supply chain with respect to Paretoequilibrium.

\section{Conflict of Interests}

The authors declare that there is no conflict of interests regarding the publication of this paper.

\section{References}

[1] I. Bose and P. Anand, "On returns policies with exogenous price," European Journal of Operational Research, vol. 178, no. 3, pp. 782-788, 2007.

[2] B. A. Pasternack, "Optimal pricing and return policies for perishable commodities," Marketing Science, vol. 27, no. 1, pp. 133140,2008 .

[3] G. P. Cachon, "Supply Chain Coordination with Contracts," Handbooks in Operations Research and Management Science, vol. 11, no. C, pp. 227-340, 2003.

[4] G. P. Cachon and M. A. Lariviere, "Supply chain coordination with revenue-sharing contracts: strengths and limitations," Management Science, vol. 51, no. 1, pp. 30-44, 2005.

[5] M. A. Lariviere, "Supply chain contracting and coordination with stochastic demand," in Quantitative Models for Supply Chain Management, pp. 233-268, Springer, 1999.

[6] N. C. Petruzzi and M. Dada, "Pricing and the newsvendor problem: a review with extensions," Operations Research, vol. 47, no. 2, pp. 183-194, 1999.

[7] C. A. Yano and S. M. Gilbert, "Coordinated pricing and production/procurement decisions: a review," in Managing Business Interfaces, pp. 65-103, Springer, 2004.

[8] Y. He, X. Zhao, L. Zhao, and J. He, "Coordinating a supply chain with effort and price dependent stochastic demand," Applied Mathematical Modelling, vol. 33, no. 6, pp. 2777-2790, 2009.

[9] H. Emmons and S. M. Gilbert, "Note: the role of returns policies in pricing and inventory decisions for catalogue goods," Management Science, vol. 44, no. 2, pp. 276-283, 1998.

[10] A. H.-L. Lau and H.-S. Lau, "The effects of reducing demand uncertainty in a manufacturer-retailer channel for singleperiod products," Computers \& Operations Research, vol. 29, no. 11, pp. 1583-1602, 2002.

[11] Z. Yao, S. C. H. Leung, and K. K. Lai, "Analysis of the impact of price-sensitivity factors on the returns policy in coordinating supply chain," European Journal of Operational Research, vol. 187, no. 1, pp. 275-282, 2008.

[12] F. J. Arcelus, S. Kumar, and G. Srinivasan, "Evaluating manufacturer's buyback policies in a single-period two-echelon framework under price-dependent stochastic demand," Omega, vol. 36, no. 5, pp. 808-824, 2008.

[13] Y. Wang, L. Jiang, and Z.-J. Shen, "Channel performance under consignment contract with revenue sharing," Management Science, vol. 50, no. 1, pp. 34-47, 2004.

[14] S. Li and Z. Hua, "A note on channel performance under consignment contract with revenue sharing," European Journal of Operational Research, vol. 184, no. 2, pp. 793-796, 2008.

[15] M. Xu, Q. Wang, and L. Ouyang, "Coordinating contracts for two-stage fashion supply chain with risk-averse retailer and price-dependent demand," Mathematical Problems in Engineering, vol. 2013, Article ID 259164, 12 pages, 2013.

[16] E. S. Mills, "Uncertainty and price theory," The Quarterly Journal of Economics, vol. 73, no. 1, pp. 116-130, 1959.

[17] S. Karlin and C. R. Carr, "Prices and optimal inventory policy," in Studies in Applied Probability and Management Science, pp. 159-172, Stanford University Press, Stanford, Calif, USA, 1962.

[18] M. A. Lariviere and E. L. Porteus, "Selling to the newsvendor: an analysis of price-only contracts," Manufacturing and Service Operations Management, vol. 3, no. 4, pp. 293-305, 2001.

[19] G. T. Thowsen, "A dynamic, nonstationary inventory problem for a price/quantity setting firm," Naval Research Logistics Quarterly, vol. 22, no. 3, pp. 461-476, 1975.

[20] J. Nash, “The bargaining problem," Econometrica, vol. 18, pp. $155-162,1950$. 


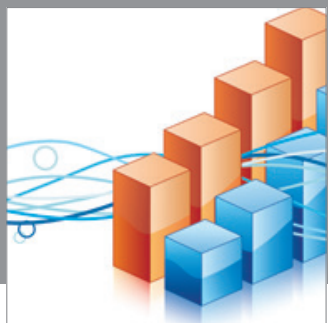

Advances in

Operations Research

mansans

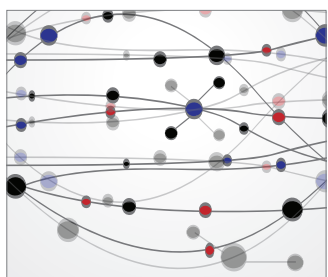

The Scientific World Journal
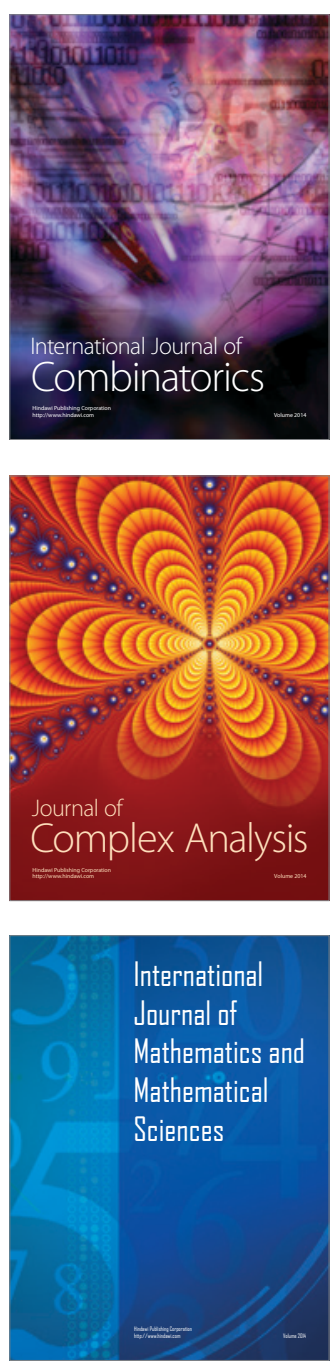
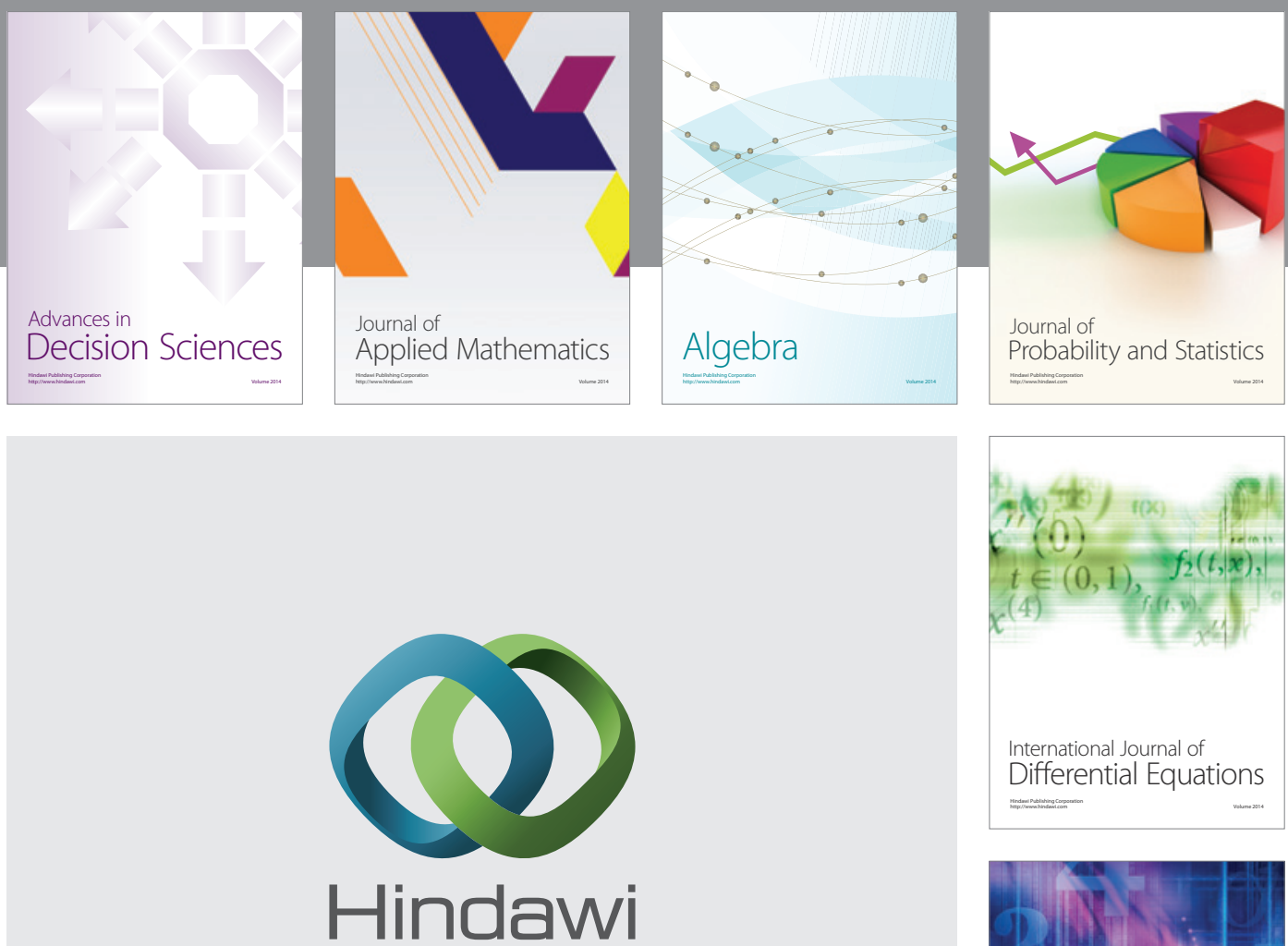

Submit your manuscripts at http://www.hindawi.com
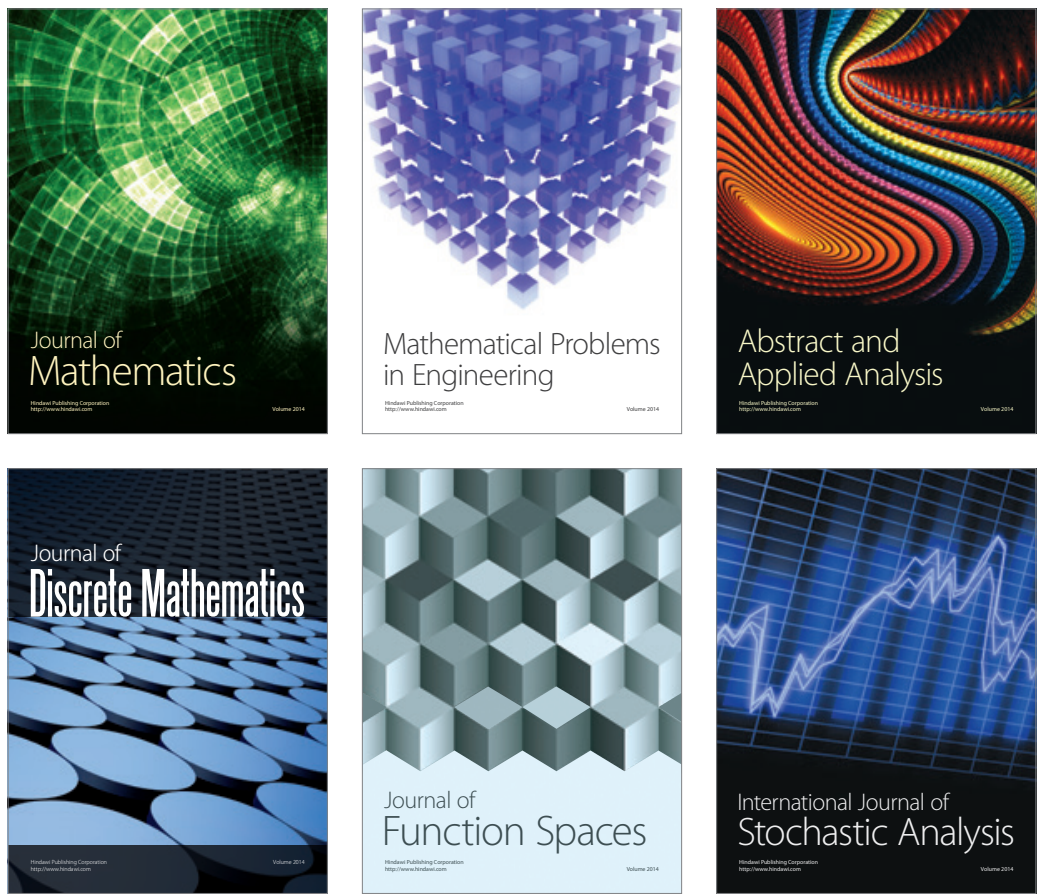

Journal of

Function Spaces

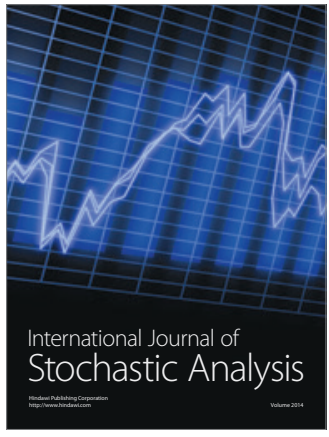

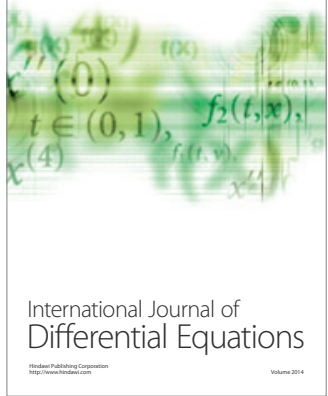
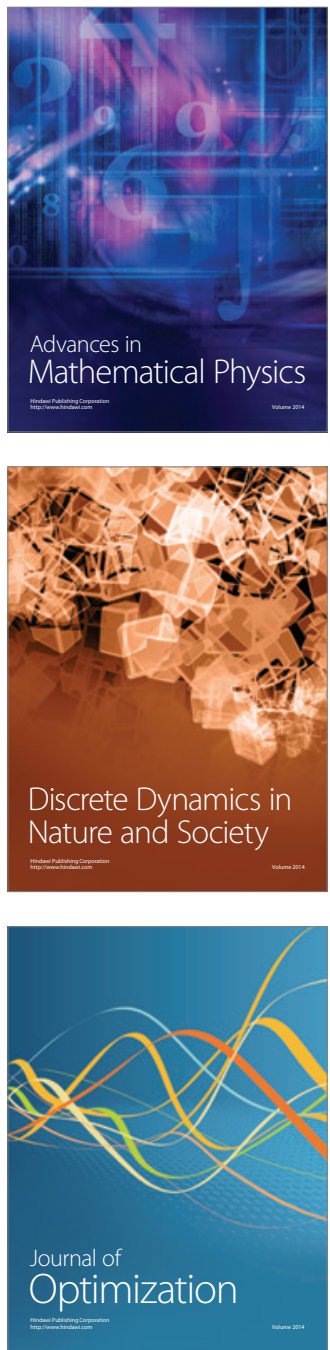